\title{
High-Efficiency, Broadband, Near Diffraction-Limited, Dielectric Metalens in Ultraviolet Spectrum
}

\author{
Saima Kanwal ${ }^{1}$, Jing Wen ${ }^{1, *}$, Binbin Yu ${ }^{1}$, Dileep Kumar ${ }^{2}$, Xu Chen ${ }^{1}$, Yi Kang ${ }^{1}$, Chunyan Bai ${ }^{1}$ \\ and Dawei Zhang ${ }^{1,3, *}$ \\ 1 Engineering Research Center of Optical Instrument and Systems, Ministry of Education and Shanghai Key \\ Lab of Modern Optical System, University of Shanghai for Science and Technology, No. 516 Jun Gong Road, \\ Shanghai 200093, China; 142499021@st.usst.edu.cn (S.K.); 161390013@st.usst.edu.cn (B.Y.); \\ 191380026@st.usst.edu.cn (X.C.); ky930827@sina.com (Y.K.); baichunyan1984@163.com (C.B.) \\ 2 State Key Laboratory of Industrial Control Technology, College of Control Science and Engineering, Zhejiang \\ University, Hangzhou 310027, China; dk2kes21@gmail.com \\ 3 Shanghai Institute of Intelligent Science and Technology, Tongji University, Shanghai 200082, China \\ * Correspondence: jwen@usst.edu.cn (J.W.); dwzhang@usst.edu.cn (D.Z.)
}

Received: 31 January 2020; Accepted: 27 February 2020; Published: 9 March 2020

\begin{abstract}
Ultraviolet (UV) optical devices have plenteous applications in the fields of nanofabrication, military, medical, sterilization, and others. Traditional optical components utilize gradual phase accumulation phenomena to alter the wave-front of the light, making them bulky, expensive, and inefficient. A dielectric metasurface could provide an auspicious approach to precisely control the amplitude, phase, and polarization of the incident light by abrupt, discrete phase changing with high efficiency due to low absorption losses. Metalenses, being one of the most attainable applications of metasurfaces, can extremely reduce the size and complexity of the optical systems. We present the design of a high-efficiency transmissive UV metalens operating in a broadband range of UV light (250-400 nm) with outstanding focusing characteristics. The polarization conversion efficiency of the nano-rod unit and the focusing efficiency of the metasurface are optimized to be as high as $96 \%$ and $77 \%$, respectively. The off-axis focusing characteristics at different incident angles are also investigated. The designed metalens that is composed of silicon nitride nanorods will significantly uphold the advancement of UV photonic devices and can provide opportunities for the miniaturization and integration of the UV nanophotonics and its applications.
\end{abstract}

Keywords: UV; metalens; broadband; diffraction-limited; PB phase

\section{Introduction}

Metasurfaces have stirred up a spree of research interest in recent years due to their brilliant performance in the field of electromagnetic wave manipulation [1-9]. Metasurfaces are based on some well-designed subwavelength scale arrays of resonators to manipulate the amplitude, phase, propagation direction, and polarization of light to nanoscale resolution at an ease [10-19], making them an appropriate option for miniaturization and integration of photonic systems. Currently, metasurfaces are being applied to various applications, such as metalenses [20], holograms [21,22], cloaking [23,24], surface plasmon launcher [4], nonlinear devices [3,11,25-28], bio sensing [26,27], computing [11,28], switching [5,29], and various novel photonic systems and devices [30-34]. Among these, metalenses are a stirring and significant research direction and application of metasurfaces since they not only outperform the optical properties but are also far smaller and ultrathin comparing to the conventional expensive and bulky optical lenses [35]. Furthermore, they provide a doorway to the integration and miniaturization of the optical devices. Transmissive or reflective metalenses can be explored by 
dielectric or plasmonic metasurfaces. Plasmonic metalenses perform well in reflection mode. However, owing to the higher reflection and absorption losses of the metals, plasmonic metalenses are less efficient in much preferred 'transmissive mode' for most of the optical devices and systems. The quest for highly efficient photonic devices has led to the employment of dielectric material as the most promising candidate for the progression of high efficiency transmissive metalenses. Optical losses are minimized to a significant extent by choosing a wide bandgap and high refractive index dielectric material. Thus, dielectric metalenses outperform the plasmonic metalenses in terms of transmission applications [36]. The desired characteristics of dielectric metalenses comprise high diffraction and transmission efficiencies, large numerical aperture (NA), and broadband wavelength operation [37].

Previously reported work on metalenses has focused on various wavelengths ranging from ultraviolet to near-infrared [38], but these metalenses defocused at a small range of UV spectrum due to the insufficient phase delay. Phase manipulation can be realized by various methods depending on principles; for instance, Huygens metasurfaces [39,40], surface plasmon wave-guiding [41,42] and dielectric effective refracting $[43,44]$. For the Pancharatnam-Berry (PB) phase method, the extra local phases of unit cells are controlled by incident angles by element rotation, and they manipulate circularly polarized (CP) light with great ability.

As mentioned above, earlier reported work on the metalenses in the UV range is lean and they do not operate in a broadband UV spectrum, and hence are defocused on a small UV range. Moreover, the focusing efficiency and the polarization conversion efficiency of the unit cell is not as high as reported in our work $[38,45,46]$. Our design of a 2-D, dielectric UV metalens is based on silicon nitride $\mathrm{Si}_{3} \mathrm{~N}_{4}$ metasurface, exhibiting high efficiency with a full phase delay of $2 \pi$ for the broadband UV range (250-400 nm) using PB phase. Our designed metalens has polarization conversion efficiency as high as $96 \%$, thus enabling ultra-high focusing efficiency to $77 \%$. Furthermore, the calculated full width at half maximum (FWHMs) of the focal spot is also diffraction limited. We envision that the designed metalens will open up a new doorway towards the development of miniaturization and integration of the UV photonic devices and its applications.

\section{Materials and Methods}

The capability to realize high efficiency dielectric metalens in the targeted UV region is critically based on the optical properties of the material, as indicated by the complex refractive index, $\tilde{n}=n+i k$. The adopted material should have a high refractive index $(n>2)$ with relatively negligible absorption loss $(k \approx 0)$ at the operating UV regime. Despite a negligible absorption being crucial for high transmission efficiency, a higher refractive index assures strong confinement of the UV light that ultimately provide full ( 0 to $2 \pi$ ) phase control [47]. The bandgap of traditional dielectric materials is narrow, causing high absorption losses in UV. Gallium nitride and titanium oxide have relatively large bandgaps but they are not appropriate to use in the targeted UV spectrum due to their higher absorption losses [46]. $\mathrm{Si}_{3} \mathrm{~N}_{4}$ has been chosen as the dielectric material for its high refractive index of about 2.3, an ultra-wide bandgap of about $5.1 \mathrm{eV}$, and its transparency window $(k \approx 0)$ for the UV spectrum of 250-400 nm [48]. Phase manipulation can be achieved through various methods relying on principles such as surface plasmon wave-guiding [41,42], dielectric effective refracting [43,44], and metamaterial Huygens surfaces $[39,40]$. However, the PB phase method has gained significant attention for being wavelength-independent $[49,50]$. In this approach, all the building blocks of the metasurface have identical size and uniformly transmitted amplitude. When the circularly polarized light is converted into inverse circular polarized light, the transmitted inversed polarized light will have a geometric phase shift that is double of the rotating angle of the nanorod. The metalens design presented here is based on high aspect ratio $\mathrm{Si}_{3} \mathrm{~N}_{4}$ nanorods as shown in Figure 1. 


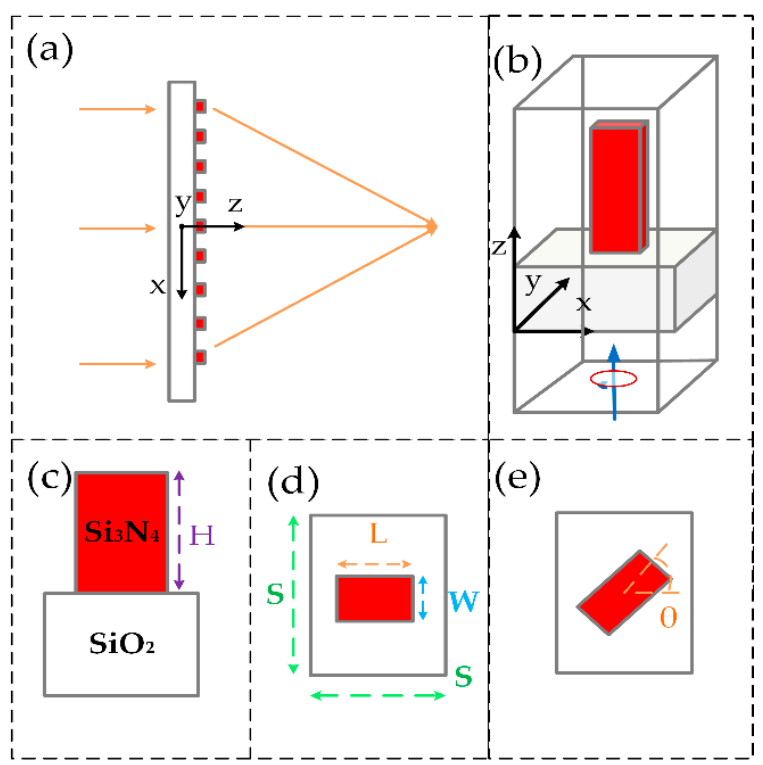

Figure 1. Design of the metalens. (a) Schematic of the metalens and its building block, the $\mathrm{Si}_{3} \mathrm{~N}_{4}$ nanorod. (b) $\mathrm{Si}_{3} \mathrm{~N}_{4}$ nanorod on $\mathrm{SiO}_{2}$ substrate. (c) and (d) side and top views of the unit cell showing the height, width, and length of the nanorod having unit cell dimensions $S \times S$. (e) By the rotation of the nanorod, the required phase is imparted by an angle of $\theta$ following the geometric Panchratnam-Berry phase.

The incident circularly polarized light can be partly converted into inversed circularly polarized light that has the geometric phase according to the PB phase method. In our design, the incident light was left circularly polarized light (LCP) and the transmitted light was right circularly polarized light (RCP). In case of incident LCP light, for a nanorod with rotating angle $\theta$ the generated phase shift $\varphi$ will be $\varphi=2 \theta$. All the simulations were performed using the commercial finite-difference-time-domain (FDTD) method implemented by commercial software 'FDTD Solutions'(produced by Lumerical Solutions Co. Ltd., Vancouver, BC, Canada) [51]. For the polarization conversion efficiency calculation, periodic boundary conditions were applied to both $\mathrm{x}$ and $\mathrm{y}$-direction and perfectly matched layer (PML) is applied to the $\mathrm{z}$-direction. The simulation area was discretized by a 3-D grid mesh using a step size of $0.25 \mathrm{~nm}$ in $\mathrm{x}, \mathrm{y}$, and $\mathrm{z}$-directions.

Polarization conversion efficiency can be defined as the fraction of the optical power of the incident circular polarized light which is converted to the optical power of the transmitted inversed circular polarized light [46,51]. By carefully optimizing the unit cell parameters such as height, width, length and period of the nanorod maximum polarization conversion efficiency can be attained. The conversion efficiency of the $\mathrm{Si}_{3} \mathrm{~N}_{4}$ nanorod in the wavelength range of $250-400 \mathrm{~nm}$ is shown in Figure 2 . 

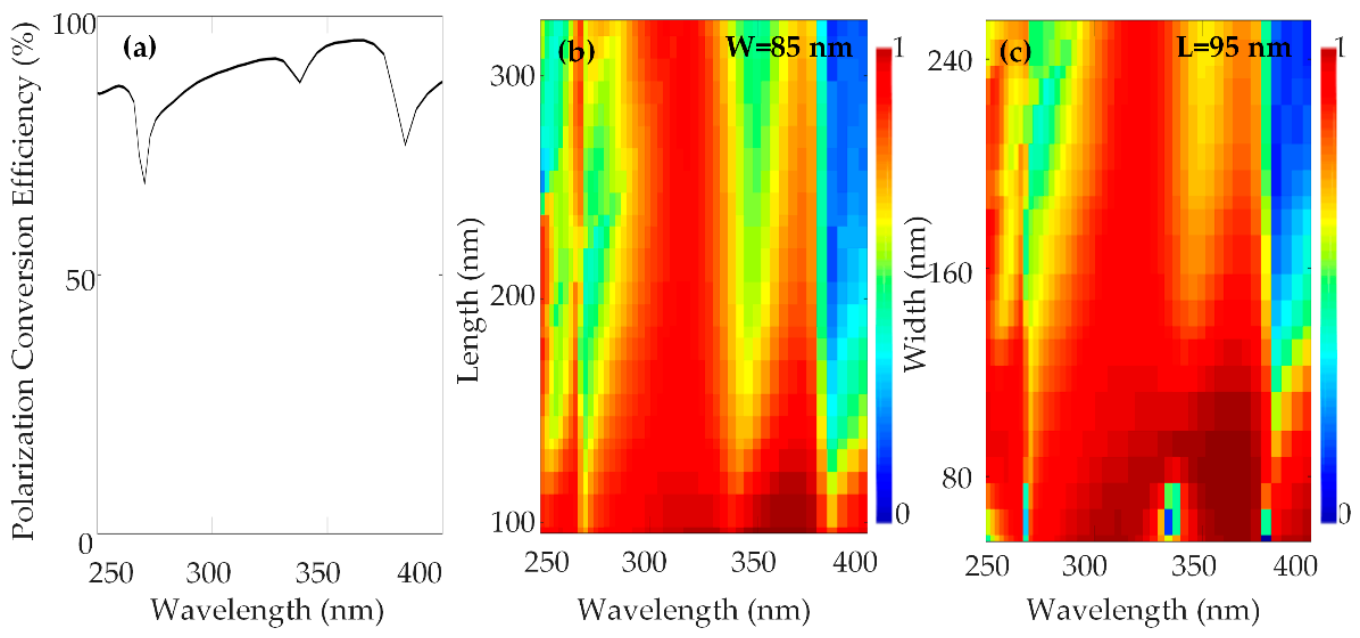

Figure 2. (a) Simulated polarization conversion efficiency as a function of wavelength for which periodic boundary conditions were applied in $\mathrm{x}$ and $\mathrm{y}$ directions and perfectly matched layer boundary conditions were applied in the z-direction. (b) Polarization conversion efficiency as a function of wavelength and length of the nanorod at width, $W=85 \mathrm{~nm}$ (c) Polarization conversion efficiency as a function of the wavelength and width of the nanorod at length, $\mathrm{L}=95 \mathrm{~nm}$.

The polarization conversion efficiency is as high as $96 \%$. The optimized structure parameters for the unit cell are height $\mathrm{H}=210 \mathrm{~nm}$, width $\mathrm{W}=85 \mathrm{~nm}$, length $\mathrm{L}=95 \mathrm{~nm}$, and period $\mathrm{S} \times \mathrm{S}=240 \mathrm{~nm}$.

\section{Design of the Metalens, Results, and Discussion}

PB phase method was implemented for designing the broadband UV metalens for light convergence at a broadband range of UV light (i.e., $250-400 \mathrm{~nm}$ ). The incident light with the wavelength $\lambda$ is focused to a spot by phase control. For a traditional spherical lens, the difference of refractive index between the different media generates the phase shift. As the light propagates through the surface of metalens to the focal point, different positions in the metalens lead to different optical path difference which has a phase shift relative to the center of the metalens (i.e., [51-53]).

$$
\varphi(x, y)=\frac{2 \pi}{\lambda}\left(f-\sqrt{f^{2}+x^{2}+y^{2}}\right)
$$

where, $x$ and $y$ are the coordinates of the nanorod in the metalens, $f$ is the focal length and $\lambda$ is the wavelength. As the wavelength and the focal length have been determined, the phase $\varphi(x, y)$ for each nanorod can be calculated. This phase profile is imparted by the rotation of each nanorod at a given coordinate $(x, y)$ by an angle $\theta(x, y)$. According to the Pancharatnam-Berry phase method, the phase shift generated by the rotation of the nanorod at the $(x, y)$ position of the metalens has a relationship as [51-53]

$$
\varphi(x, y)=2 \theta(x, y)
$$

where, $\theta(x, y)$ is the rotating angle of the nanorod in the position $(x, y)$, hence each nanorod in the metalens is rotated by an angle of [51-53]

$$
\theta(x, y)=\frac{\pi}{\lambda}\left(f-\sqrt{f^{2}+x^{2}+y^{2}}\right)
$$


According to the above equation, the rotation angle of all the nanorods is determined at each position. It should be noted that the rotation angle can be an arbitrary value from 0 to $\pi$, so the optimized nanorod structure could be able to alter the phase profile of transmitted light at will. Therefore, the transmitted PB phase can achieve full 0 to $2 \pi$ phase range. The optimized structure parameters for the designed metalens in the wavelength range 250-400 $\mathrm{nm}$ were chosen as height $\mathrm{H}=340 \mathrm{~nm}$, width $\mathrm{W}=85 \mathrm{~nm}$, length $\mathrm{L}=150 \mathrm{~nm}$, and period $\mathrm{S} \times \mathrm{S}=240 \mathrm{~nm}$. The diameter of the metalens was $10 \mu \mathrm{m}$. The NA of the metalens at the design wavelength $\lambda_{d}=300 \mathrm{~nm}$ was calculated to be 0.75 . While focusing, efficiency of the metalens was as high as $77 \%$. Figure 3 presents the focusing characteristics of the designed metalens.
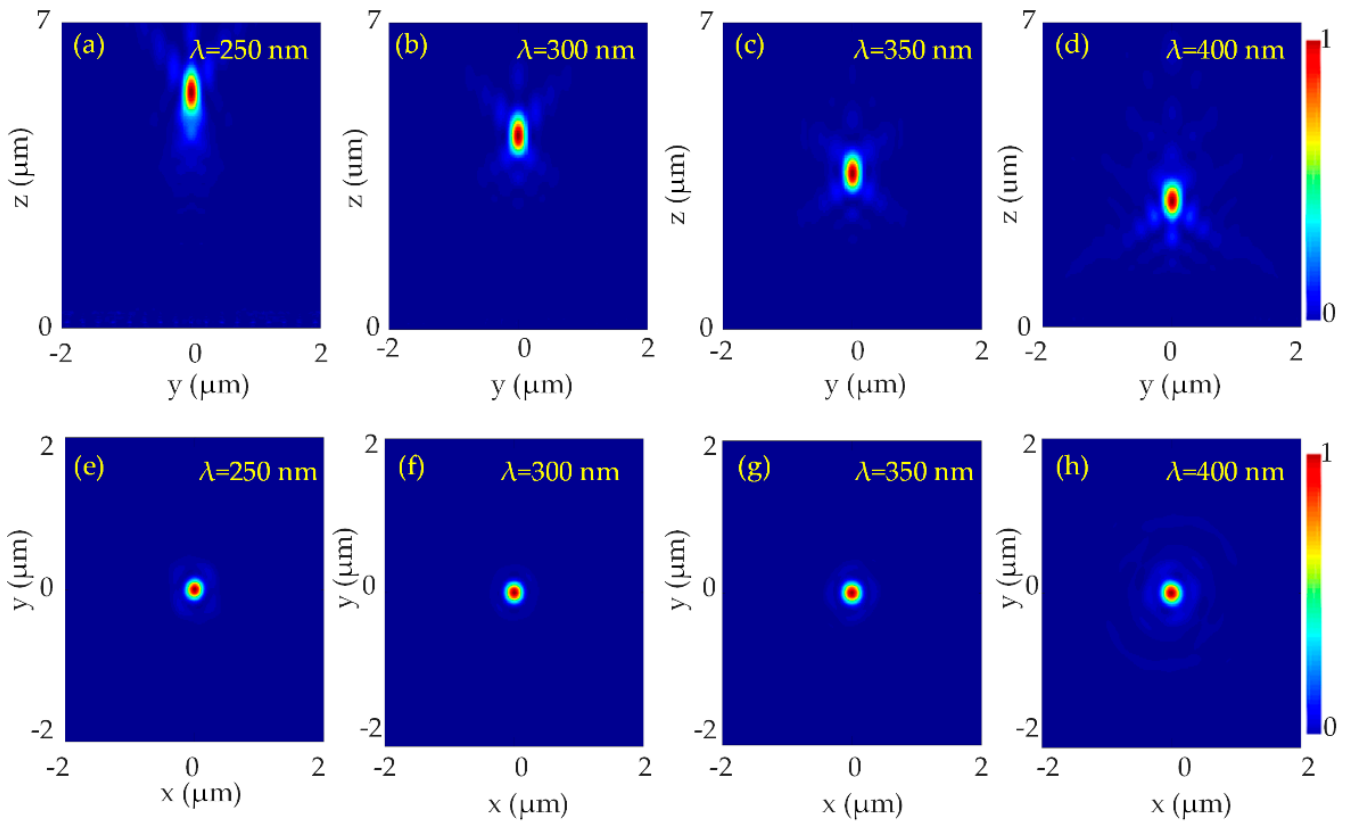

Figure 3. Focusing characteristics of UV metalense at incident UV light $250-400 \mathrm{~nm}$. Normalized intensity distribution at $y-z$ plane at (a) $\lambda=250 \mathrm{~nm},(\mathbf{b}) \lambda=300 \mathrm{~nm},(\mathbf{c}) \lambda=350 \mathrm{~nm}$ and (d) $\lambda=400 \mathrm{~nm}$. Normalized intensity distribution of the metalens at $x-y$ plane at $x=y=0$. (e) $\lambda=250 \mathrm{~nm}$ along $x$-axis at $\mathrm{z}=5.3 \mu \mathrm{m},(\mathrm{f}) \lambda=300 \mathrm{~nm}$, along $\mathrm{x}$-axis at $\mathrm{z}=4.4 \mu \mathrm{m}(\mathrm{g}) \lambda=350 \mathrm{~nm}$, along $\mathrm{x}$-axis at $\mathrm{z}=3.5 \mu \mathrm{m}$ and $(\mathbf{h}) \lambda=400 \mathrm{~nm}$, along $x$-axis at $z=2.9 \mu \mathrm{m}$. The metalens has a diameter of $10 \mu \mathrm{m}$, the numerical aperture (NA) of the metalens at designed wavelength $\lambda_{d}=300 \mathrm{~nm}$ is 0.75 .

The normalized intensity distributions of the transmitted light beam in the $y-z$ section of the metalens are shown in Figure $3 a-d$ and the intensity profiles of the transmitted light beam in the $x-y$ section of the metalens are shown in Figure $3 e-h$. The intensity distribution of the focal spot exhibits a strongly focused, bright, and symmetric spot at the center of the focal plane at respective wavelengths $(250 \mathrm{~nm}, 300 \mathrm{~nm}, 350 \mathrm{~nm}$, and $400 \mathrm{~nm})$. The corresponding normalized intensity profiles along the $\mathrm{x}$-axis show a sharp peak at the center of the plane as shown in Figure 4. 

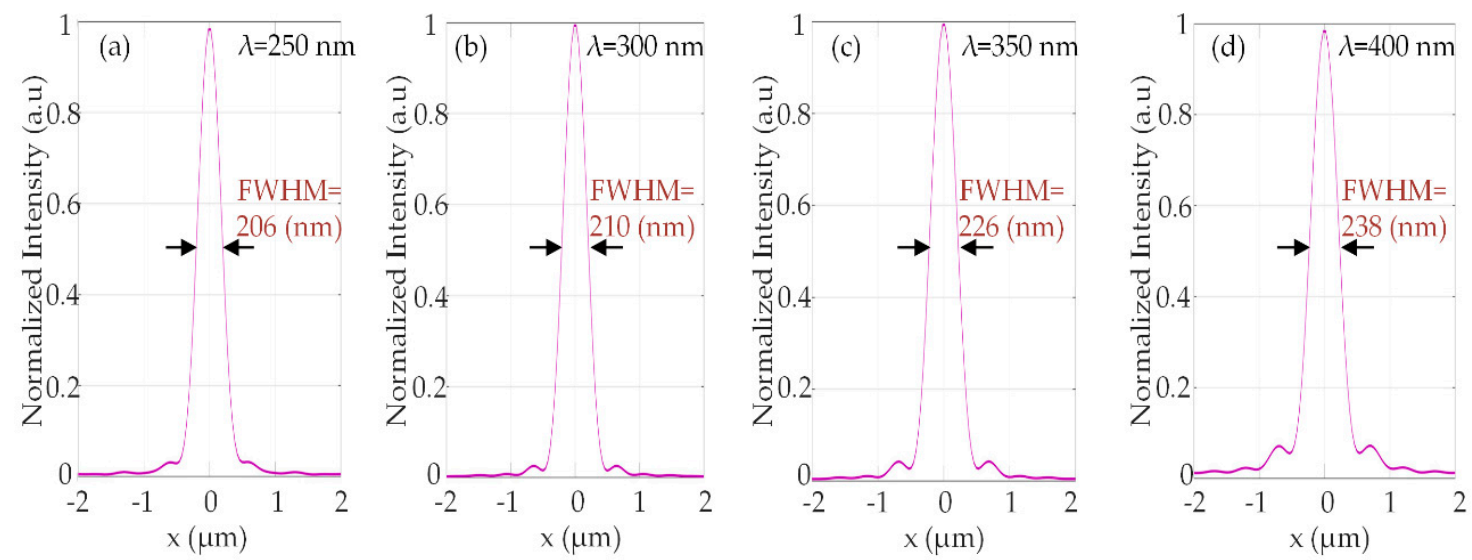

Figure 4. Normalized intensity profiles of the focus spot along $x$-direction at respective wavelengths (a) $\mathrm{y}=0, \lambda=250 \mathrm{~nm}, \mathrm{z}=5.3 \mu \mathrm{m},(\mathbf{b}) \mathrm{y}=0, \lambda=300 \mathrm{~nm}, \mathrm{z}=4.4 \mu \mathrm{m}$, (c) $\mathrm{y}=0, \lambda=350 \mathrm{~nm}, \mathrm{z}=3.5 \mu \mathrm{m}$ and (d) $\mathrm{y}=0, \lambda=400 \mathrm{~nm}, \mathrm{z}=2.9 \mu \mathrm{m}$.

The focal length of the metalens was measured at representative wavelengths $(250 \mathrm{~nm}, 300 \mathrm{~nm}$, $350 \mathrm{~nm}$, and $400 \mathrm{~nm}$ ). The calculated full width at half maximum (FWHM) of the focal spots at the respective wavelengths was $206 \mathrm{~nm}, 210 \mathrm{~nm}, 226 \mathrm{~nm}$, and $238 \mathrm{~nm}$; all the values were diffraction-limited (i.e., $\frac{\lambda_{d}}{2 N A}$ ). The normalized intensity profiles of the focal spot along $x$-direction (at $z=f$, and $y=0$ ) are shown in Figure 4 . The focusing efficiency of the UV metalens is measured to be as high as $77 \%$, shown in Figure 5.

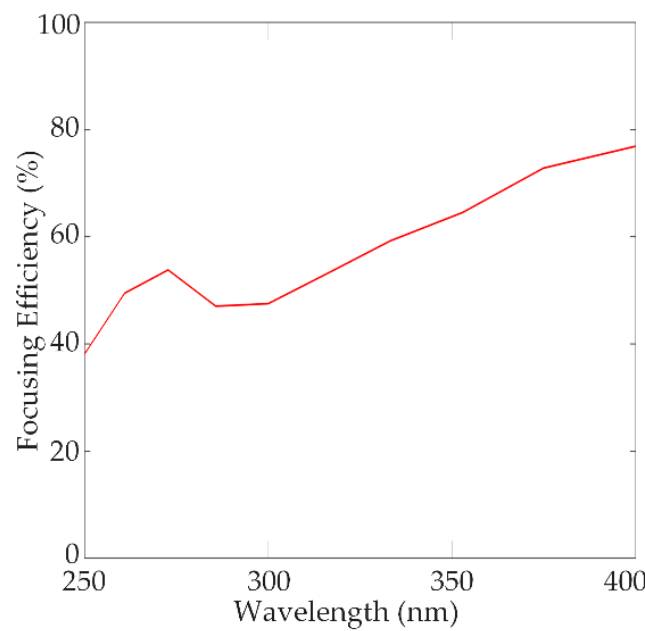

Figure 5. Focusing efficiency of the metalens designed for the broadband UV spectrum 250-400 nm.

We verified four wavelengths during the optimizing process; the designed metalens worked quite well over the broadband range of $250-400 \mathrm{~nm}$.

We also examined the focusing characteristics of the designed metalens at different incident angles (i.e., $5^{\circ}, 15^{\circ}$, and $25^{\circ}$ ). It can be seen by the simulation results presented in Figure 6 that the transmitted light beam was clear. Figure 6 shows the focusing characteristics and corresponding normalized intensity distribution of the focal spot at different incident angles at the respective wavelengths. 

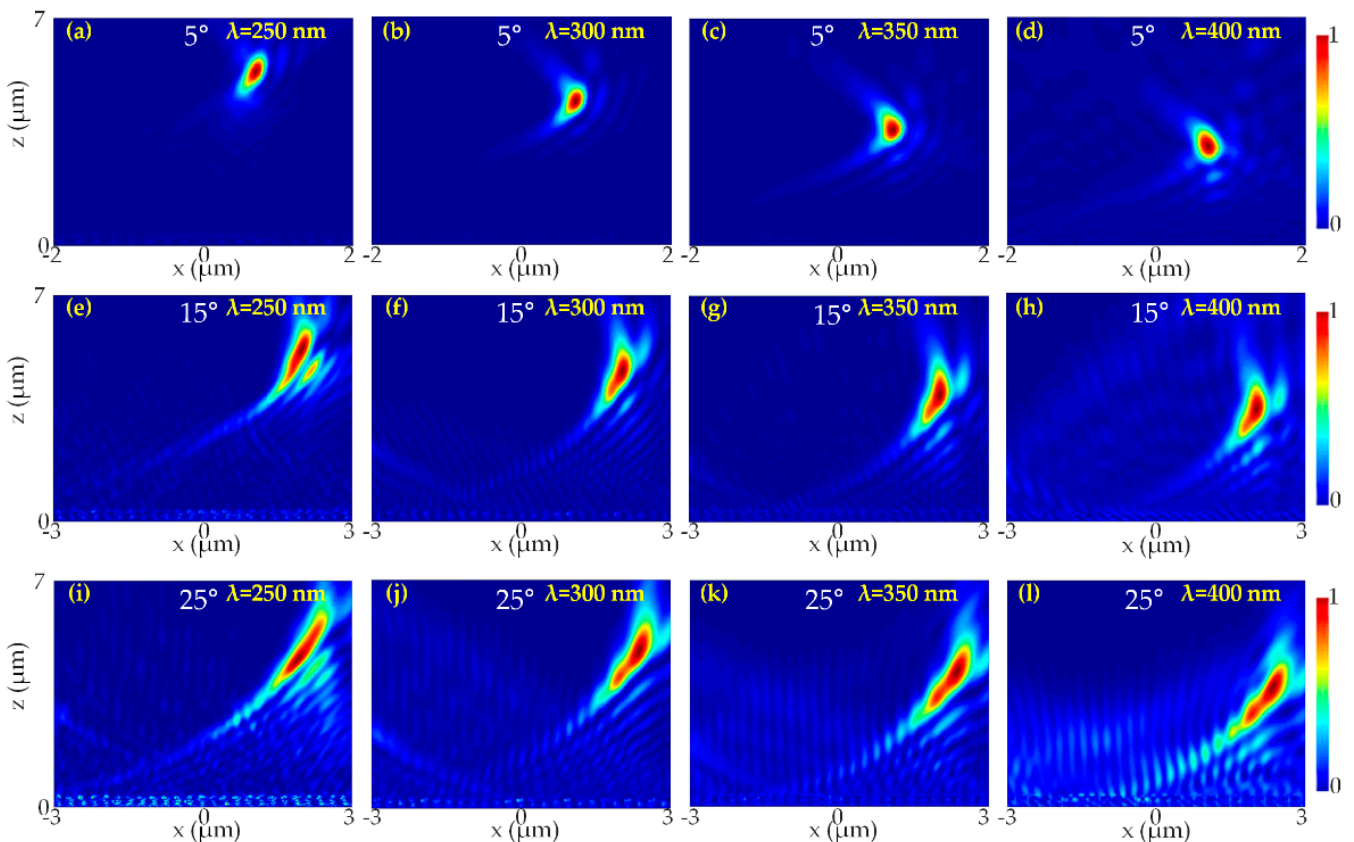

Figure 6. Focusing characteristics of UV metalens at 250-400 $\mathrm{nm}$ at different incident angles. Normalized intensity distribution at $x-z$ plane at an incident angle of $5^{\circ}(\mathbf{a}) \lambda=250 \mathrm{~nm},(\mathbf{b}) \lambda=300 \mathrm{~nm},(\mathbf{c}) \lambda=350 \mathrm{~nm}$ and (d) $\lambda=400 \mathrm{~nm}$. Normalized intensity distribution of the metalens at $\mathrm{x}-\mathrm{z}$ plane at an incident angle of $15^{\circ}(\mathbf{e}) \lambda=250 \mathrm{~nm},(\mathbf{f}) \lambda=300 \mathrm{~nm}(\mathrm{~g}) \lambda=350 \mathrm{~nm}$ and $(\mathbf{h}) \lambda=400 \mathrm{~nm}$. Normalized intensity distribution at $\mathrm{x}-\mathrm{z}$ plane at an incident angle of $25^{\circ}$ (i) $\lambda=250 \mathrm{~nm}(\mathbf{j}) \lambda=300 \mathrm{~nm}(\mathbf{k}) \lambda=350 \mathrm{~nm}$ and (1) $\lambda=400 \mathrm{~nm}$

\section{Conclusions}

In summary, we report the design of a dielectric, high efficiency, diffraction-limited UV metalens based on silicon nitride metasurface functioning in the broadband spectrum of ultraviolet light (i.e., from 250-400 nm). PB phase was implemented to the unit cell to realize the required phase distribution of the metalens. The simulated conversion efficiency was $96 \%$ while the focusing efficiency of the metalens was as high as $77 \%$. We also investigated the focusing characteristics of the metalens at different incident angles (i.e., $5^{\circ}, 15^{\circ}$, and $25^{\circ}$ ), which showed a clear focus spot. The designed UV metalens can have a great promising perception for a diverse range of applications in lithography, UV laser, UV directional light, image sensors, sterilization, communication, and so on. This research on high efficiency broadband UV metalenses can pave the way towards the advancement of miniaturized and integrated UV nanophotonics. Techniques like multiple couple resonances, tailoring the phase profiles at numerous distinct UV wavelengths, engineering the dispersion, or stacking metasurfaces could be adopted in the future for designing an achromatic UV metalens.

Author Contributions: S.K. conceived and designed the experiments, and wrote the original manuscript; B.Y., D.K., X.C., Y.K., and C.B. contributed to the analysis; J.W. and D.Z. supervised the study and reviewed the manuscript. All authors have read and agreed to the published version of the manuscript.

Funding: This work is supported by National Science Foundation Project 61775140 and 81701745, National Key R\&D Program of China 2018YFA0701800, Shanghai Municipal Science and Technology Commission Innovation Action Plan 18DZ1100400.

Conflicts of Interest: The authors declare no conflict of interest.

\section{References}

1. Mueller, J.B.; Rubin, N.A.; Devlin, R.C.; Groever, B.; Capasso, F. Metasurface polarization optics: Independent phase control of arbitrary orthogonal states of polarization. Phys. Rev. Lett. 2017, 118, 113901. [CrossRef] [PubMed] 
2. Khorasaninejad, M.; Shi, Z.; Zhu, A.Y.; Chen, W.-T.; Sanjeev, V.; Zaidi, A.; Capasso, F. Achromatic metalens over $60 \mathrm{~nm}$ bandwidth in the visible and metalens with reverse chromatic dispersion. Nano Lett. 2017, 17, 1819-1824. [CrossRef] [PubMed]

3. Li, G.; Zhang, S.; Zentgraf, T. Nonlinear photonic metasurfaces. Nat. Rev. Mater. 2017, 2, 1-14. [CrossRef]

4. Jiang, Q.; Bao, Y.; Lin, F.; Zhu, X.; Zhang, S.; Fang, Z. Spin-controlled integrated near-and far-field optical launcher. Adv. Funct. Mater. 2018, 28, 1705503. [CrossRef]

5. Ahmadivand, A.; Gerislioglu, B.; Ramezani, Z. Gated graphene island-enabled tunable charge transfer plasmon terahertz metamodulator. Nanoscale 2019, 11, 8091-8095. [CrossRef]

6. Yu, B.; Wen, J.; Chen, X.; Zhang, D. An achromatic metalens in the near-infrared region with an array based on a single nano-rod unit. Appl. Phys. Express 2019, 12, 092003. [CrossRef]

7. Bai, W.; Yang, P.; Wang, S.; Huang, J.; Chen, D.; Zhang, Z.; Yang, J.; Xu, B. Tunable duplex metalens based on phase-change materials in communication range. Nanomaterials 2019, 9, 993. [CrossRef]

8. Liang, Y.; Liu, H.; Wang, F.; Meng, H.; Guo, J.; Li, J.; Wei, Z. High-efficiency, near-diffraction limited, dielectric metasurface lenses based on crystalline titanium dioxide at visible wavelengths. Nanomaterials 2018, 8, 288. [CrossRef]

9. Li, B.; Piyawattanametha, W.; Qiu, Z. Metalens-Based Miniaturized Optical Systems. Micromachines 2019, 10, 310. [CrossRef]

10. Kiarashinejad, Y.; Abdollahramezani, S.; Adibi, A. Deep learning approach based on dimensionality reduction for designing electromagnetic nanostructures. arXiv 2019, arXiv:1902.03865. [CrossRef]

11. Abdollahramezani, S.; Chizari, A.; Dorche, A.E.; Jamali, M.V.; Salehi, J.A. Dielectric metasurfaces solve differential and integro-differential equations. Opt. Lett. 2017, 42, 1197-1200. [CrossRef] [PubMed]

12. Abdollahramezani, S.; Taghinejad, H.; Fan, T.; Kiarashinejad, Y.; Eftekhar, A.A.; Adibi, A. Reconfigurable multifunctional metasurfaces employing hybrid phase-change plasmonic architecture. arXiv 2018, arXiv:1809.08907.

13. Zhang, M.; Pu, M.; Zhang, F.; Guo, Y.; He, Q.; Ma, X.; Huang, Y.; Li, X.; Yu, H.; Luo, X. Plasmonic metasurfaces for switchable photonic spin-orbit interactions based on phase change materials. Adv. Sci. 2018, 5, 1800835. [CrossRef] [PubMed]

14. De Galarreta, C.R.; Alexeev, A.M.; Au, Y.Y.; Lopez-Garcia, M.; Klemm, M.; Cryan, M.; Bertolotti, J.; Wright, C.D. Nonvolatile reconfigurable phase-change metadevices for beam steering in the near infrared. Adv. Funct. Mater. 2018, 28, 1704993. [CrossRef]

15. Dong, W.; Qiu, Y.; Zhou, X.; Banas, A.; Banas, K.; Breese, M.B.; Cao, T.; Simpson, R.E. Tunable mid-infrared phase-change metasurface. Adv. Opt. Mater. 2018, 6, 1701346. [CrossRef]

16. She, A.; Zhang, S.; Shian, S.; Clarke, D.R.; Capasso, F. Adaptive metalenses with simultaneous electrical control of focal length, astigmatism, and shift. Sci. Adv. 2018, 4, eaap9957. [CrossRef]

17. Callewaert, F.; Velev, V.; Jiang, S.; Sahakian, A.V.; Kumar, P.; Aydin, K. Inverse-designed stretchable metalens with tunable focal distance. Appl. Phys. Lett. 2018, 112, 091102. [CrossRef]

18. Ding, P.; Li, Y.; Shao, L.; Tian, X.; Wang, J.; Fan, C. Graphene aperture-based metalens for dynamic focusing of terahertz waves. Opt. Express 2018, 26, 28038-28050. [CrossRef]

19. Chen, W.T.; Zhu, A.Y.; Sanjeev, V.; Khorasaninejad, M.; Shi, Z.; Lee, E.; Capasso, F. A broadband achromatic metalens for focusing and imaging in the visible. Nat. Nanotechnol. 2018, 13, 220-226. [CrossRef]

20. Fu, R.; Li, Z.; Zheng, G.; Chen, M.; Yang, Y.; Tao, J.; Wu, L.; Deng, Q. Reconfigurable step-zoom metalens without optical and mechanical compensations. Opt. Express 2019, 27, 12221-12230. [CrossRef]

21. Zheng, G.; Mühlenbernd, H.; Kenney, M.; Li, G.; Zentgraf, T.; Zhang, S. Metasurface holograms reaching 80\% efficiency. Nat. Nanotechnol. 2015, 10, 308-312. [CrossRef] [PubMed]

22. Wang, L.; Kruk, S.; Tang, H.; Li, T.; Kravchenko, I.; Neshev, D.N.; Kivshar, Y.S. Grayscale transparent metasurface holograms. Optica 2016, 3, 1504-1505. [CrossRef]

23. Ni, X.; Wong, Z.J.; Mrejen, M.; Wang, Y.; Zhang, X. An ultrathin invisibility skin cloak for visible light. Science 2015, 349, 1310-1314. [CrossRef] [PubMed]

24. Yang, Y.; Jing, L.; Zheng, B.; Hao, R.; Yin, W.; Li, E.; Soukoulis, C.M.; Chen, H. Full-polarization 3D metasurface cloak with preserved amplitude and phase. Adv. Mater. 2016, 28, 6866-6871. [CrossRef] [PubMed]

25. Lee, J.; Tymchenko, M.; Argyropoulos, C.; Chen, P.-Y.; Lu, F.; Demmerle, F.; Boehm, G.; Amann, M.-C.; Alu, A.; Belkin, M.A. Giant nonlinear response from plasmonic metasurfaces coupled to intersubband transitions. Nature 2014, 511, 65-69. [CrossRef] 
26. Jakšić, Z.; Vuković, S.; Matovic, J.; Tanasković, D. Negative refractive index metasurfaces for enhanced biosensing. Materials 2011, 4, 1-36. [CrossRef]

27. Ahmadivand, A.; Gerislioglu, B.; Manickam, P.; Kaushik, A.; Bhansali, S.; Nair, M.; Pala, N. Rapid detection of infectious envelope proteins by magnetoplasmonic toroidal metasensors. ACS Sens. 2017, 2, 1359-1368. [CrossRef]

28. Chizari, A.; Abdollahramezani, S.; Jamali, M.V.; Salehi, J.A. Analog optical computing based on a dielectric meta-reflect array. Opt. Lett. 2016, 41, 3451-3454. [CrossRef]

29. Buchnev, O.; Podoliak, N.; Kaczmarek, M.; Zheludev, N.I.; Fedotov, V.A. Electrically controlled nanostructured metasurface loaded with liquid crystal: Toward multifunctional photonic switch. Adv. Opt. Mater. 2015, 3, 674-679. [CrossRef]

30. Chen, C.C.; Ishikawa, A.; Tang, Y.H.; Shiao, M.H.; Tsai, D.P.; Tanaka, T. Uniaxial-isotropic Metamaterials by Three-Dimensional Split-Ring Resonators. Adv. Opt. Mater. 2015, 3, 44-48. [CrossRef]

31. Tittl, A.; Harats, M.G.; Walter, R.; Yin, X.; Schäferling, M.; Liu, N.; Rapaport, R.; Giessen, H. Quantitative angle-resolved small-spot reflectance measurements on plasmonic perfect absorbers: Impedance matching and disorder effects. Acs Nano 2014, 8, 10885-10892. [CrossRef] [PubMed]

32. Gerislioglu, B.; Dong, L.; Ahmadivand, A.; Hu, H.; Nordlander, P.; Halas, N.J. Monolithic metal dimer-on-film structure: New plasmonic properties introduced by the underlying metal. Nano Lett. 2020. [CrossRef] [PubMed]

33. Gerislioglu, B.; Ahmadivand, A. Functional Charge Transfer Plasmon Metadevices. Research 2020, 2020, 9468692. [CrossRef] [PubMed]

34. Ahmadivand, A.; Semmlinger, M.; Dong, L.; Gerislioglu, B.; Nordlander, P.; Halas, N.J. Toroidal dipole-enhanced third harmonic generation of deep ultraviolet light using plasmonic meta-atoms. Nano Lett. 2018, 19, 605-611. [CrossRef] [PubMed]

35. Bai, W.; Yang, P.; Huang, J.; Chen, D.; Zhang, J.; Zhang, Z.; Yang, J.; Xu, B. Near-infrared tunable metalens based on phase change material Ge 2 Se 2 Te 5. Sci. Rep. 2019, 9, 1-9. [CrossRef] [PubMed]

36. Choudhury, S.M.; Wang, D.; Chaudhuri, K.; DeVault, C.; Kildishev, A.V.; Boltasseva, A.; Shalaev, V.M. Material platforms for optical metasurfaces. Nanophotonics 2018, 7, 959-987. [CrossRef]

37. Ozdemir, A. High Efficient Ultra-Thin Flat Optics Based on Dielectric Metasurfaces. Ph.D. Thesis, The University of Arizona, Tucson, AZ, USA, 2018.

38. Ollanik, A.J.; Smith, J.A.; Belue, M.J.; Escarra, M.D. High-efficiency all-dielectric Huygens metasurfaces from the ultraviolet to the infrared. ACS Photonics 2018, 5, 1351-1358. [CrossRef]

39. Pfeiffer, C.; Emani, N.K.; Shaltout, A.M.; Boltasseva, A.; Shalaev, V.M.; Grbic, A. Efficient light bending with isotropic metamaterial Huygens' surfaces. Nano Lett. 2014, 14, 2491-2497. [CrossRef]

40. Pfeiffer, C.; Grbic, A. Metamaterial Huygens' surfaces: Tailoring wave fronts with reflectionless sheets. Phys. Rev. Lett. 2013, 110, 197401. [CrossRef]

41. Xu, T.; Du, C.; Wang, C.; Luo, X. Subwavelength imaging by metallic slab lens with nanoslits. Appl. Phys. Lett. 2007, 91, 201501. [CrossRef]

42. Luo, X. Principles of electromagnetic waves in metasurfaces. Sci. China Phys. Mech. Astron. 2015, 58, 594201. [CrossRef]

43. Khorasaninejad, M.; Zhu, A.Y.; Roques-Carmes, C.; Chen, W.T.; Oh, J.; Mishra, I.; Devlin, R.C.; Capasso, F. Polarization-insensitive metalenses at visible wavelengths. Nano Lett. 2016, 16, 7229-7234. [CrossRef] [PubMed]

44. Shi, Z.; Khorasaninejad, M.; Huang, Y.-W.; Roques-Carmes, C.; Zhu, A.Y.; Chen, W.T.; Sanjeev, V.; Ding, Z.-W.; Tamagnone, M.; Chaudhary, K. Single-layer metasurface with controllable multiwavelength functions. Nano Lett. 2018, 18, 2420-2427. [CrossRef] [PubMed]

45. Guo, L.; Xu, S.; Wan, R.; Li, T.; Xiong, L.; Wang, L.; Zhu, W. Design of aluminum nitride metalens in the ultraviolet spectrum. J. Nanophotonics 2018, 12, 043513.

46. Guo, L.; Hu, Z.; Wan, R.; Long, L.; Li, T.; Yan, J.; Lin, Y.; Zhang, L.; Zhu, W.; Wang, L. Design of aluminum nitride metalens for broadband ultraviolet incidence routing. Nanophotonics 2018, 8, 171-180. [CrossRef]

47. Devlin, R.C.; Khorasaninejad, M.; Chen, W.T.; Oh, J.; Capasso, F. Broadband high-efficiency dielectric metasurfaces for the visible spectrum. Proc. Natl. Acad. Sci. USA 2016, 113, 10473-10478. [CrossRef] [PubMed] 
48. Palik, E.D.; Ghosh, G. Electronic Handbook of Optical Constants of Solids: User Guide; Academic Press: Cambridge, MA, USA, 1999.

49. Pancharatnam, S. Generalized theory of interference and its applications. Proc. Indian Acad. Sci. Sect. A 1979, 88, 398-417. [CrossRef]

50. Berry, M.V. Quantal phase factors accompanying adiabatic changes. Proc. R. Soc. Lond. A Math. Phys. Sci. 1984, 392, 45-57.

51. Khorasaninejad, M.; Chen, W.T.; Devlin, R.C.; Oh, J.; Zhu, A.Y.; Capasso, F. Metalenses at visible wavelengths: Diffraction-limited focusing and subwavelength resolution imaging. Science 2016, 352, 1190-1194. [CrossRef]

52. Dong, H.; Wang, F.; Liang, R.; Wei, Z.; Meng, H.; Jiang, L.-H.; Cen, H.; Wang, L.; Qin, S.; Wang, C. Visible-wavelength metalenses for diffraction-limited focusing of double polarization and vortex beams. Opt. Mater. Express 2017, 7, 4029-4037. [CrossRef]

53. Yang, H.; Li, G.; Su, X.; Cao, G.; Zhao, Z.; Chen, X.; Lu, W. Reflective metalens with sub-diffraction-limited and multifunctional focusing. Sci. Rep. 2017, 7, 1-10. [CrossRef] [PubMed]

(C) 2020 by the authors. Licensee MDPI, Basel, Switzerland. This article is an open access article distributed under the terms and conditions of the Creative Commons Attribution (CC BY) license (http://creativecommons.org/licenses/by/4.0/). 